\title{
Comparison Analysis of Flame Sources, Loss Quantities and Atmospheric Conditions of Kerosene and Gas in Domestic and Industrial Heating Processes
}

\author{
Casmir Onyeneke ${ }^{1}$, Rich Ndubuisi ${ }^{1}$, Edward Victorhez ${ }^{2}$, Isaac Ayetuoma ${ }^{2}$ \\ ${ }^{1}$ Department of Mathematics and Statistics, Hezekiah University, Umudi, Nigeria \\ ${ }^{2}$ Department of Computer Science, Hezekiah University, Umudi, Nigeria
}

Email address:

edwardvictorhez@gmail.com (E. Victorhez), casdyx $a$ yahoo.com (C. Onyeneke)

\section{To cite this article:}

Edward Victorhez, Casimir Onyeneke, Isaac Ayetuoma, Rich Ndubuisi. Comparison Analysis of Flame Sources, Loss Quantities and Atmospheric Conditions of Kerosene and Gas in Domestic and Industrial Heating Processes. Biomedical Statistics and Informatics. Vol. 3, No. 2, 2018, pp. 15-21. doi: 10.11648/j.bsi.20180302.12

Received: July 2, 2018; Accepted: July 13, 2018; Published: August 9, 2018

\begin{abstract}
Gas and kerosene are the two major sources of fuel used at home and industrial cooking. The process of optimizing these resources poses great challenge to industrial cooking and heating. This work examined whether gas and kerosene are rapidly exhausted when heat is applied at high temperature to heating cylinder and stove respectively. At the end, reliable and efficient conditions that optimize heating process are proffered. It was also observed that the rate of decrease in the substances used while cooking depends on the quantity of heat applied at various temperatures. In both domestic and industrial heating processes, there is a major concern of the appropriate fuel source and atmospheric conditions to be adopted. This research work presented the costs and effects of adopting a particular method. It is obvious that there is a particular cost and benefit attached to each method. This work analyzed them to enable a concise and suitable choice of heating process. At the end, there was clear indication that gas sustains cooking than kerosene when heat is applied.
\end{abstract}

Keywords: Industrial Cooking, Temperature, Orthogonal Comparison, Loss Quantity, Atmospheric Conditions

\section{Introduction}

Industrial and domestic cooking is predominantly done with cooking gas and kerosene. These substances undergo series of serious purification before they are used as source of fuel. It is not enough to enhance cooking without the presence of light, which converts mechanical energy to heat and light energy. In another development, cooking takes place ineffectively without the presence of an enhanced air. It is among the essential factors that enhance the constant lightening and production of desired heating flame. Though air contains 73 percent nitrogen, 21 percent oxygen, 0.03 percent carbon dioxide and 0.01 percent inert gases [1], the most critical element is oxygen because light needs it to enhance constant production of flame and heat. Effective industrial heating takes place when all the required environmental and atmospheric conditions necessary to boost flames are considered.

According to Brownian motion gas molecules undergo random motion [2]. If heat is applied to gas, its kinetic energy is increased and transferring heat environment through exothermic reaction. The rate which gas molecules move in a container is affected by the amount of heat applied to it. The tendency of gas molecules to spread fast, release heat to its environment due to their molecular kinetic energy and the distance created by the molecules makes it preferable in industrial and domestic cooking. In the other hand, we have kerosene as a source of energy in stoves. And this source needs to be transported from the trunk of the stove to the burner through wool [3]. Wools have the capacity of assimilating liquids gradually from trough to crest. It does not burn concurrently in absence of kerosene because the presence of kerosene makes it lighter to flame well. Wools drain the kerosene gradually to the top where combustion takes place and sustain a free constant flame with the aid of air. As the wool burns its size is reduced vertically resulting to the idea of increasing the wool by adjusting or tuning the stove jack.

The size of the wool determines the amount of kerosene 
convey to the crest [4]. Wool of $2 \mathrm{~mm}$ by $5 \mathrm{~cm}$ transfers a little quantity of kerosene decreasing the quantity of flame produced while wool of $4 \mathrm{~mm}$ by $5 \mathrm{cmproduces}$ more flame and heat. As wool brings kerosene to stove acme, the kerosene molecules heat and evaporate which in turn reduces the quantity in the container. At normal circumstance, especially in a hot weather condition the rate by which kerosene evaporates varies from the evaporation that takes place in water and gasoline. Evaporation in kerosene is not often noticeable, because of its molecular nature. During the process of heating, kerosene loses molecules to the environment [5]. The quality of flame produced depends on purity level of kerosene and size of the wool. Red-like flames are undesirable due to black smoke it produces. This is mostly as a result of combustion wool undergo with the purified substance containing thin paraffin and alkane hydrocarbon called kerosene. It is not all the quantity of flame produced during wool heating is useful. Some are converted to smoke causing black ashes while others are converted to full heat enhancing effective boiling. Kerosene are easily ecstatic because they are not like gas molecules which are always in random motion [6], and are likely to retain its content in a material like wool.

\section{Methodology}

The experiments were conducted and observed at various conditions. In regards to air, flame was introduced to 8 pieces of wood in a ventilated area. The same type and quantity of wood were flame and placed in a blocked area where air cannot penetrate. A burning burner with 1 litre of kerosene, 12 pieces of wools of $2 \mathrm{~mm}$ by $5 \mathrm{~cm}$ were well fixed in the burner and flame under non-ventilated condition. The similar experiment was set in a ventilated area. In two other different set ups, $4 \mathrm{~mm}$ by $5 \mathrm{~cm}$ of wool were fixed to the burner and flame with and without ventilation respectively. At this point, the presence of sunlight was introduced to a burner with ventilation having the same materials such as 1 litre of kerosene, 12 wools of $4 \mathrm{~mm}$ by $5 \mathrm{~cm}$. The same set up was made at this point and placed in a cold area where the air is moist.

In other cases, four different gas cylinders of $6 \mathrm{~kg}$ content each were placed in ventilated, sunlight, cold, non-ventilated areas respectively and set aflame. After that, a burner of 6 litres of kerosene, 12 pieces of thread wools of $4 \mathrm{~mm}$ by $5 \mathrm{~cm}$ was set on flame where there is ventilation, cold, hot air and without ventilation and observed in different set ups according to their atmospheric conditions. Here, a burning stove burner of $30 \mathrm{ml}$ of kerosene, 12 wools of $4 \mathrm{~mm}$ by $5 \mathrm{~cm}$ each was set under sunlight and observed. After that, a stove filled with same amount and size of wool but 1 litre of kerosene was increased times three and placed in a ventilated area. Furthermore, the same materials were placed in a ventilated area, but the jack was turned to the highest level. At the end, a cylinder filled with 6 litres of kerosene with wool of $4 \mathrm{~mm}$ by $5 \mathrm{~cm}$ was set in a ventilated area. A stove with fuse $6 \mathrm{~kg}$ of gas molecules and wool of $4 \mathrm{~mm}$ by $5 \mathrm{~cm}$ was placed in a ventilated atmospheric condition. The entire arrangement was presented in Table 1 .

Table 1. Presentation of Experimental Conditions.

\begin{tabular}{lll}
\hline Method & Materials & Conditions Required \\
\hline 1 & 8 pieces of wood, light. & In presence of air/ ventilation. \\
2 & 8 pieces of wood, light. & Absence of air/ no ventilation. \\
3 & Stove, 1 litre of kerosene, 12 wools of any seize, light. & Absence air/ no ventilation. \\
4 & Stove, 1 litre of kerosene, 12 wools of any size, light. & Presence of air/ventilation. \\
5 & Stove, 1 litre of kerosene with 12 wools of $4 \mathrm{~mm}$ by $5 \mathrm{~cm}$, light. & No ventilation. \\
6 & Stove, 1 litre of kerosene, with 12 wools of $4 \mathrm{~mm}$ by $5 \mathrm{~cm}$, light. & Ventilation. \\
7 & Stove, I litre of kerosene, with 12 wools of $4 \mathrm{~mm}$ by $5 \mathrm{~cm}$, light. & Sunlight. \\
8 & Stove, I litre of kerosene with 12 wools of $4 \mathrm{~mm}$ by $5 \mathrm{~cm}$, light. & Cold \\
9 & Gas cylinder, $6 \mathrm{~kg}$ of gas molecules, light & No ventilation \\
10 & Gas cylinder, $6 \mathrm{~kg}$ of gas molecules, light & Presence of air \\
11 & Gas cylinder, $6 \mathrm{~kg}$ of gas molecules, light. & Sunlight $/$ hot area. \\
12 & Gas cylinder, $6 \mathrm{~kg}$ of gas molecules, light.. & Cold area. \\
13 & Stove, 6 litres of kerosene, 12 wools of $4 \mathrm{~mm}$ by $5 \mathrm{~cm}$, light. & Presence of air. \\
14 & Stove, 6 litres of fuel, wool of $4 \mathrm{~mm}$ by $5 \mathrm{~cm}$, light. & Cold. \\
15 & Stove, 6 litres of fuel, wool of $4 \mathrm{~mm}$ by $5 \mathrm{~cm}$, light. & Sunlight. \\
16 & Stove, 6 litres of pure water, wool of $4 \mathrm{~mm}$ by $5 \mathrm{~cm}$, light. & Ventilation. \\
17 & Stove, $30 \mathrm{ml}$ of kerosene $\mathrm{s}$ & Sunlight $/$ hot area. \\
18 & Stove, 1 litre of kerosene, 12 wools of $4 \mathrm{~mm}$ by $5 \mathrm{~cm}$ (turning the jack 3 times), light. & Ventilation. \\
19 & Stove, 1 litre of kerosene, 12 wools of $4 \mathrm{~mm}$ by $5 \mathrm{~cm}$ (turning the jack to the highest), light. & Ventilation. \\
20 & Cylinder, 6 litres of kerosene, wool of $4 \mathrm{~mm}$ by $5 \mathrm{~cm}$, light. & Ventilation. \\
21 & Stove, $6 \mathrm{~kg}$ of gas content, pipe $/$ channel, light. & Ventilation. \\
\hline
\end{tabular}

\section{Result and Discussion}

In the grounds of this study, presence of air makes woods to burn faster affirming that oxygen supports combustion and the rate in which flame increase is high, producing big amount of heat [7]. It was discovered that the high amount of air resulted to the high rate of combustion in a ventilated case. In absence of air, huge amount of heat was produced causing the blue flame unstable, and it generate much smoke, which is chocking when inhaled. This type flame does not enhance cooking, rather it negatively affect industrial heating 
and cooking. In a ventilated experimental set up, quantity of the flame increased, the heat generated is higher than that of $2 \mathrm{~mm}$ by $5 \mathrm{~cm}$ wools and blue flame was produced. The degree in which food boils here is higher with less smoke [8]. When $4 \mathrm{~mm}$ by $5 \mathrm{~cm}$ wools was used, it produced non-blue flame. This experiment delays cooking, but maintains conducive state of health and atmosphere.

Under the condition of no air, a non-blue coloured flame was produced, and a lot of heat was observed. In the presence of sunlight, blue flame was produced. The utensil heated faster and the quantity of the kerosene decreased gradually than the set up in absence of sunlight. Placing the stove under a cool condition, the blue flame was produced, conducting less smoke [9]. The utensil heated faster and approaches the rate of the set up under sunlight condition. In the absence of air, unstable blue flame was produced, and the smell remained dominant in that area. In presence of oxygen, stable blue flame was produced and the smell was not easily perceived. The rate of heat generated is high [10].

Placing the cylinder under sunlight/hot area, gas molecules were set in a great motion and a higher blue flame was produced. The $6 \mathrm{~kg}$ of gas molecules are highly affected by the great motion caused by the additional heat, sourced by sunlight. Blue light was produced. Smell was perceived [11]. The gas movement was not as high as result in the presence of ventilation, food substances tends to boil not fast as we have result when oxygen was introduced. Under a cool condition, the wools burned faster, yellow light was produced, which has a great tendency of exploding while sunlight condition makes yellow light produced with tendency to explode faster than result in cold condition [12]. The $30 \mathrm{ml}$ of kerosene remained $29.3 \mathrm{ml}$ after 5 hours; it was observed that quantity loss quantity was $0.7 \mathrm{ml}$. In other words, $0.7 \mathrm{ml}$ was lost under sunlight within 5 hours showing the rate of evaporation. In the event of 10 hours, the amount of kerosene to be evaporated is $10 / 5 \times 0.7 / 1=1.4 \mathrm{ml}$. When the jack was increase 3 times, blue flame was produced, enhancing less smoke. Cooking utensil boiled gradually without staining the back of the pot. High flame was produced in ventilated condition, which has yellow colour mixed with blue. The cooking utensil boils more than the normal case, but the utensil was highly stained with black smokes. Changing the content in cylinder to kerosene with wool produced blue flame but the rate in which the food boiled was slow [13]. When the content of stove was changed to gas and placed outside the house, constant flame was not retained, rather the gas reacted to the light by exploding and some gases escaped immediately due to the openings.

Table 2. Presentation of Observations and Inferences.

\begin{tabular}{|c|c|c|}
\hline Method & Observation & Inference \\
\hline 1 & Wood burns faster increasing the Heat. & Air supports combustion \\
\hline 2 & Heat generated, Unstable Flame. & Reduction in combustion \\
\hline 3 & Insufficient flame produced, smoke, Heat. & No ventilation \\
\hline 4 & Hard to generate light, little kerosene conveyed & Oxygen supports combustion \\
\hline 5 & Flame size increased, much heat generated plus blue flame. & Ventilation Contributes to heating \\
\hline 6 & $4 \mathrm{~mm}$ by $5 \mathrm{~cm}$ wool produces a non-blue flame. & No Ventilation \\
\hline 7 & Blue flame, less smoke, rapid heating and decrease in kerosene. & Sunlight/Hot Area \\
\hline 9 & Blue flame plus dominant smell. & No Ventilation \\
\hline 10 & Blue flame plus mere smell with increased heat and flame. & Ventilation \\
\hline 11 & Better blue flame with great motion and much heat & Sunlight/Hot Area. \\
\hline 12 & Blue flame, increased in motion faster cooking. & $\ldots \ldots \ldots \ldots$ \\
\hline 13 & Occurrence of method 12 result. & \\
\hline 14 & Wools burned faster with yellow flame and explosive tendency. & Cool Area. \\
\hline 15 & Yellow light, more explosive than method 14. & Sunlight. \\
\hline 16 & Loss of $0.7 \mathrm{ml}$ within 5 hours, greater evaporation. & Sunlight enhances evaporation \\
\hline 18 & High mixture of yellow and blue flame plus smokes and stains. & In Presence Of Air. \\
\hline 19 & Black smoke with slow heating & Gas heats faster than kerosene \\
\hline 20 & Loss of heat, gas molecule explosion and lack of constant flame & Excessive exposure affects heating \\
\hline
\end{tabular}

Table 3. Data Representation.

\begin{tabular}{|c|c|c|c|c|c|c|}
\hline Method S/N Method & No of Stove & Wool size (mm:cm) & Wool No & Jack Turn Decree & No of $\operatorname{wood}(L:$ W) each & Seize of wood $(\mathrm{cm})$ \\
\hline 1 & - & - & - & - & 8 & $5: 2$ \\
\hline 2 & - & - & - & - & 8 & $5: 2$ \\
\hline 3 & 1 & $2: 5$ & 12 & 1440 & - & - \\
\hline 4 & 1 & $4: 5$ & 12 & 1440 & - & - \\
\hline 5 & 1 & $4: 5$ & 12 & 1440 & - & - \\
\hline 6 & 1 & $4: 5$ & 12 & 1440 & - & - \\
\hline 8 & 1 & 0 & - & - & - & - \\
\hline 9 & - & 0 & - & - & - & - \\
\hline 10 & - & 0 & - & - & - & - \\
\hline
\end{tabular}




\begin{tabular}{lllllll}
\hline Method S/N Method & No of Stove & Wool size (mm:cm) & Wool No & Jack Turn Decree & No of wood (L: W) each & Seize of wood (cm) \\
\hline 11 & - & 0 & - & - & - & - \\
12 & - & $4: 5$ & 12 & 1440 & - & - \\
13 & - & $4: 5$ & 12 & 1440 & - & - \\
14 & 1 & $4: 5$ & 12 & 1440 & - & - \\
15 & 1 & $4: 5$ & 12 & 1440 & - & - \\
16 & 1 & $4: 5$ & 12 & 1440 & - & - \\
17 & 1 & $4: 5$ & 12 & 1440 & - & - \\
18 & 1 & $4: 5$ & 12 & 1080 & - & - \\
19 & 1 & $4: 5$ & - & - & - & - \\
20 & 0 & - & 1 & - & - \\
\hline
\end{tabular}

Table 3. Continued.

\begin{tabular}{llllll}
\hline No of cylinder & Quan. of fuel & Quan. of kerosene & Quan. of H20 & Quan. of gas & Turn of the cylinder decree \\
\hline- & - & - & - & - & - \\
- & - & - & - & - & - \\
- & - & 1 & - & - & - \\
- & - & 1 & - & - & - \\
- & - & - & - & - & - \\
- & - & 1 & - & - & 720 \\
- & - & 1 & - & 6 & 720 \\
- & - & - & - & 6 & 720 \\
- & - & - & - & 6 & - \\
- & - & - & - & 6 & - \\
- & - & - & - & - & - \\
- & - & 6 & - & - & - \\
- & - & - & - & - & - \\
- & 6 & - & 6 & - & - \\
- & 6 & - & - & - & - \\
- & - & 0.3 & - & - & - \\
- & - & 1 & - & - & - \\
\hline & - & 1 & - & 6 & 720 \\
\hline
\end{tabular}

The average time per litre taken for the different atmospheric conditions by randomly assigned cooking fuels with no prior experience of the boiling time of these fuel mechanisms after the same amount of water and instructions was observed and recorded. The primary interest here was to compare several different methods of boiling water. The cooking methods give varied boiling times. The experimental set up was carried out with 1 litre of water and the boiling time was recorded in minutes at different experimental set up shown below

Table 4. Flame Sources and Atmospheric Conditions (in Minutes).

\begin{tabular}{|c|c|c|c|c|c|c|c|}
\hline & Ventilation & Non-Ventilation & Sunlight & Cold air & $T_{i}$ & $\overline{\boldsymbol{x}}_{\boldsymbol{i}}$ & $\bar{d}_{i}$ \\
\hline Fire wood & 25 & 38 & 16 & 32 & 111 & 27.75 & -1.1875 \\
\hline Kerosene stove & 30 & 40 & 35 & 31 & 136 & 34 & 5.0625 \\
\hline Electric stove & 28 & 33 & 30 & 32 & 123 & 30.75 & 1.8125 \\
\hline Gas cooker & 15 & 30 & 20 & 28 & 93 & 23.25 & -5.6875 \\
\hline$T_{j}$ & 98 & 141 & 101 & 123 & 463 & $\downarrow$ & \multirow{2}{*}{$\downarrow$} \\
\hline $\bar{x}_{j}$ & 24.5 & 35.25 & 25.25 & 30.75 & $\rightarrow$ & 115.75 & \\
\hline $\bar{d}_{j}$ & -4.4375 & 6.3125 & -3.6875 & 1.8125 & $\rightarrow$ & & 0.0000 \\
\hline
\end{tabular}

The primary interest here is to state the model, estimate the mean effect of each composition and to ascertain at 95 percent level of confidence if there is any significance difference in the boiling methods. 


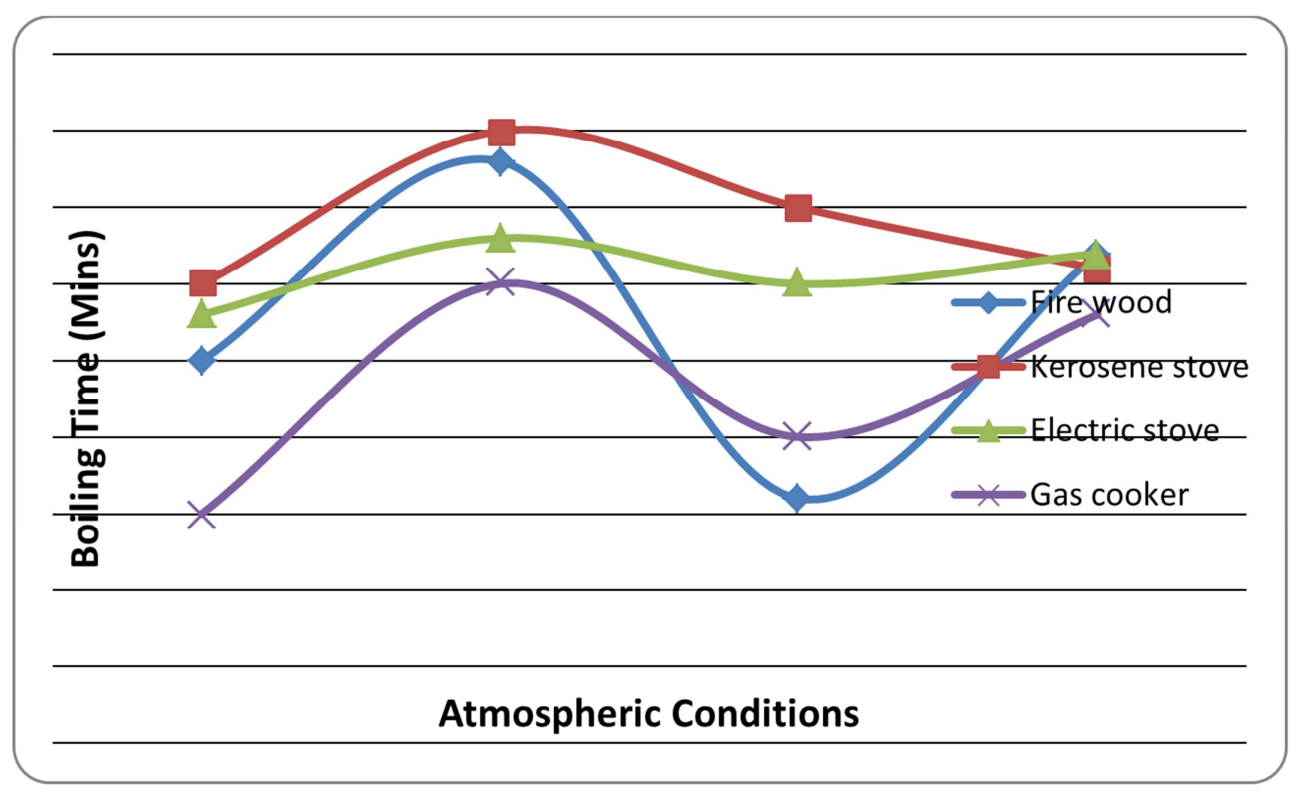

Figure 1. Flame Sources and Boiling Time.

The loss quantity for kerosene and gas were respectively recorded at different atmospheric conditions measured in mililitre.

Table 5. Correlation of Loss Quantity between Gas and Kerosene.

\begin{tabular}{|c|c|c|c|c|c|c|}
\hline \multirow{2}{*}{ Source } & \multirow{2}{*}{ Ventilation } & \multirow{2}{*}{ Non-Ventilation } & \multirow{2}{*}{ Sunlight } & \multirow{2}{*}{ Cold air } & \multicolumn{2}{|c|}{ Correlation } \\
\hline & & & & & Kerosene & Gas \\
\hline Kerosene & 0.45 & 0.65 & 0.70 & 0.60 & 1 & 0.926 \\
\hline Gas & 0.12 & 0.30 & 0.50 & 0.35 & 0.926 & 1 \\
\hline
\end{tabular}

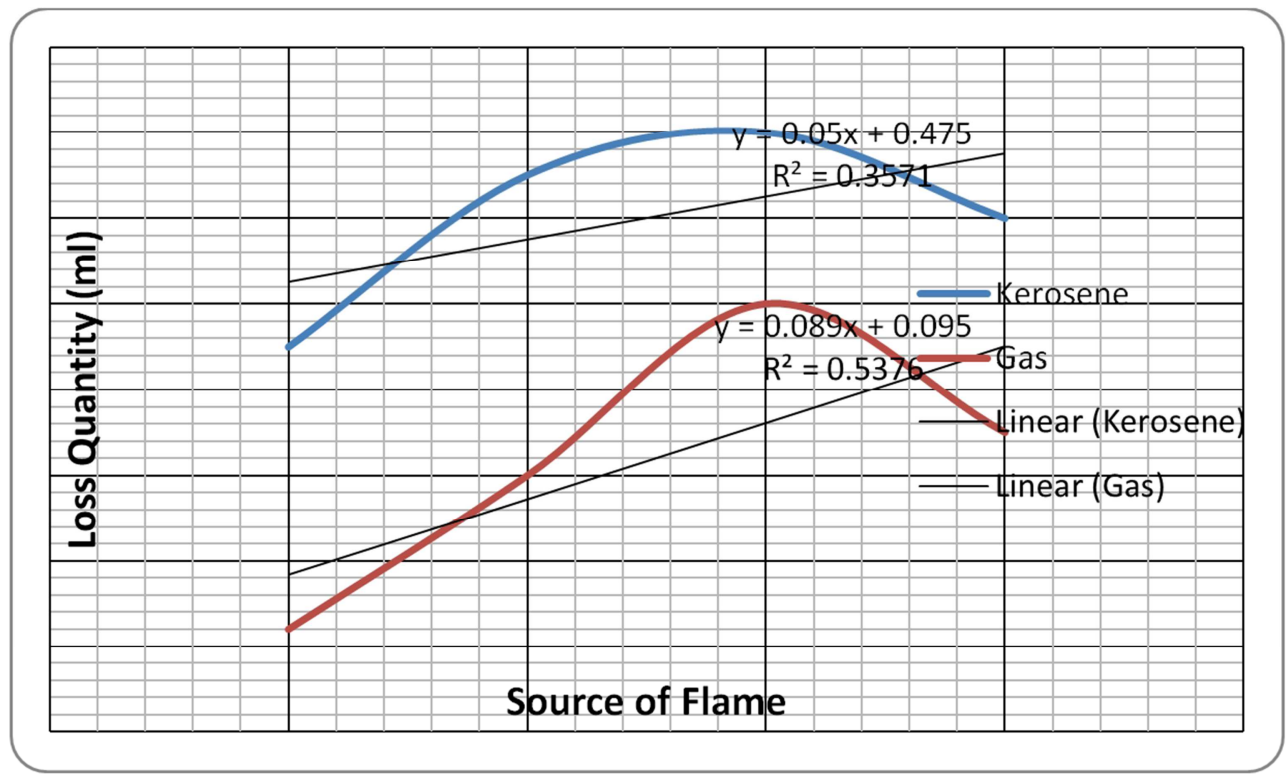

Figure 2. Comparison of Loss Quantity.

Though there is significant relationship existing between the use of gas and kerosene in heating and boiling process across the atmospheric conditions such as ventilation, sunlight, cold air and non-ventilation, it is worthy to not that they are not at exact level [14]. The analysis shows that there existed 95.3 percent relationship in the loss quantity of the two substances. In other words, one has more loss quantity than the other at a given atmospheric condition. In a general case, kerosene is 4.7 percent loss quantity greater than gas.

The model is $X_{i j}=t_{i}+b_{j}+e_{i j} ; i, j=1, \ldots, 4$ where $X_{i j}$ is the boiling time in minutes of experimental condition obtained from the $j t h$ source of fuel, $t_{i}$ is the mean effect of the fuel composition, $b_{j}$ is the mean effect of the atmospheric condition while $e_{i j}$ is the random error associated with $X_{i j}$. The analysis of variance was done and recorded. 
Table 6. Analysis of Variance.

\begin{tabular}{llllll}
\hline SSV & DF & SS & MS & F-cal & F-tab \\
\hline SSt & 3 & 250.6875 & 83.5625 & 4.03115578 & 3.86 \\
SSb & 3 & 305.688 & 101.8958 & 4.91557789 & 3.86 \\
SSe & 9 & 186.563 & 20.72917 & & \\
SST & 15 & 742.938 & & & \\
\hline
\end{tabular}

Since 4.03 is greater than 3.86 , there is enough evidence that the boiling time varies among the four fuel sources. Similarly, since 4.92 is greater than 3.86 , we also have enough evidence that the boiling atmospheric condition to atmospheric condition.

Multiple comparison tests were carried out to find out which boiling method gave the highest contribution to the variation. The mean effect of fuel source and atmospheric conditions were arranged in ascending order of magnitude as $\hat{t}_{4}=-5.6875<\hat{t}_{1}=-1.1875<\hat{t}_{3}=1.8125<\hat{t}_{2}=5.0625$ and $\hat{b}_{1}=-4.4375<\hat{b}_{3}=-3.6875<\hat{b}_{4}=1.8125<\hat{b}_{2}=$ 6.3125 respectively. The orthogonal linear comparison was set up as

Table 7. Orthogonal Comparisons.

\begin{tabular}{llllllll}
\hline \multicolumn{2}{l}{ Flame Source } & & \multicolumn{5}{c}{ Atmospheric Condition } \\
\hline$\hat{\boldsymbol{t}}_{\mathbf{1}}$ & $\hat{\boldsymbol{t}}_{\mathbf{2}}$ & $\hat{\boldsymbol{t}}_{\mathbf{3}}$ & $\hat{\boldsymbol{t}}_{\mathbf{4}}$ & $\widehat{\boldsymbol{b}}_{\mathbf{1}}$ & $\widehat{\boldsymbol{b}}_{\mathbf{2}}$ & $\widehat{\boldsymbol{b}}_{\mathbf{3}}$ & $\widehat{\boldsymbol{b}}_{\mathbf{4}}$ \\
\hline 1 & 0 & 0 & -1 & -1 & 0 & 0 & 1 \\
$-1 / 2$ & $1 / 2$ & $1 / 2$ & $-1 / 2$ & $-1 / 2$ & $1 / 2$ & $-1 / 2$ & $1 / 2$ \\
$-1 / 3$ & 1 & $-1 / 3$ & $-1 / 3$ & $-1 / 3$ & 1 & $-1 / 3$ & $-1 / 3$ \\
0 & 1 & -1 & 0 & 0 & 1 & -1 & 0 \\
\hline
\end{tabular}

Sum of squares due to the comparisons are calculated using $S S_{[i]}=\frac{\left(\sum C_{i} T_{i .}\right)^{2}}{n\left(\sum C_{i}\right)^{2}}$ and the ANOVA table is presented as

Table 8. ANOVA of the Comparisons.

\begin{tabular}{|c|c|c|c|c|c|c|c|c|c|c|c|}
\hline \multicolumn{6}{|c|}{ Fuel Source } & \multicolumn{6}{|c|}{ Atmospheric Condition } \\
\hline SSV & DF & SS & F-cal & F-tab & & SSV & DF & SS & F-cal & F-tab & \\
\hline [1] & 1 & 40.5 & 1.953765 & 5.12 & Not Sig & [1] & 1 & 78.125 & 3.768834 & 5.12 & Not Sig \\
\hline [2] & 1 & 189.0625 & 9.120582 & 5.12 & Sig & [2] & 1 & 264.0625 & 12.73866 & 5.12 & Sig \\
\hline [3] & 1 & 136.6875 & 6.593949 & 5.12 & Sig & [3] & 1 & 212.5208 & 10.25223 & 5.12 & Sig \\
\hline [4] & 1 & 21.125 & 1.019097 & 5.12 & Not Sig & [4] & 1 & 200 & 9.648215 & 5.12 & Sig \\
\hline Error & 9 & 186.563 & & & & Error & 9 & 186.563 & & & \\
\hline
\end{tabular}

The fuel sources and the atmospheric conditions were respectively checked under four comparisons. Fire wood flame is not significantly different from other sources of fuel [15]. The same case was observed when the minutes used in boiling with cold air are compared to the amount of minutes used in boiling with ventilation. There is a significant difference between the amount of minutes used in boiling water with electric stove and kerosene stove joined together with other sources. Also, the result obtained showed enough discrepancy between the times used when boiling same volume of water with kerosene stove against other sources of fuel. Comparing boiling without ventilation to other atmospheric conditions, there are significant differences [16]. However, the number of times used in boiling water with kerosene stove is significantly different from the number of minutes used in boiling same volume of water using electric stove.

\section{Conclusion}

In Industrial and domestic cooking, it is obvious that combustion and constant lightning take place if air with much content of oxygen is present. Air is an essential component while cooking or burning or doing anything that involves heating. In the absence of air, smoke is been produced and the colour of the flame becomes undesirable. It turns to yellow or mixture of yellow and blue instead of pure blue. Excess smoke effects taste of food after cooking in a non ventilated area. Hot areas or condition and the presence of sunlight make our food to boil fast than in a cold area that has ventilation also. Hot areas or hot weather condition causes evaporation in kerosene in a very little quantity compared to water or fuel. It causes increase in random movement of gas molecules causing higher reduction of gas than when placed in a cool ventilated area.

Gases can easily operate or work in a closed system and pressure increases depending on the weather condition or temperature. Gasoline in a cylinder brings about safety in cooking than practicing what was done using kerosene and stove. Kerosene is more convenient, suitable to boil in a stove than in a cylinder. Kerosene is good to use as a source of energy than water which do not conduct flame and fuel which conduct heat, but very dangerous to our health). Whether you are using gas or stove all needs a ventilated area, and the higher the amount of air the higher the light maintain its constant flame, and tends to save from hazardous smoke. It is preferable to cook in a cool ventilated area than to cook in the presence of sunlight or under the penetration of sunlight. Using gas cylinder is good and retains its source than stove.

Finally, the rate of reduction in gas and kerosene is also affected by the intensity/length or temperature or how high the flame and heat is produced. The higher the temperature the higher the level in which the kerosene dry and the higher the gas molecules moves out.

\section{References}

[1] Jewett W. J. and Serway R. A. (2008). Physics For Scientists And Engineers With Modern Physics, 7th Ed. Cenage Learning India Private Limited 418, F. I. E Patparganj Delhi 110092, India.

[2] Brooker. J. R., Widmaier E. P. \&Stiling P. D. 2008. Biology. Janice Roerige- Blong: McGraw-Hill Publishing Company.

[3] Banse, Timothy (2010). "Kerosene Outboards: An Alternative Fuel?" Marine Engine Digest. 
[4] Collins, Chris (2007). "Implementing Phytoremediation of Petroleum Hydrocarbons". Methods in Biotechnology. Humana Press (23): 99-108. ISBN 1-58829-541-9.

[5] Annamalai, Kalyan; IshwarKanwarPuri (2006). Combustion Science and Engineering. CRC Press. p. 851. ISBN 978-08493-2071-2.

[6] Feng, Lianyong; Hu, Yan; Hall, Charles A. S; Wang, Jianliang (2013). The Chinese Oil Industry: History and Future. Springer (published November 28, 2012). p. 2. ISBN 9781441994097.

[7] Chang, Samuel Hsu; Robinson, Paul R. (2006). Practical Advances in Petroleum Processing. 1. Springer. p. 2.

[8] Russell, Loris S. (2003). A Heritage of Light: Lamps and Lighting in the Early Canadian Home. University of Toronto Press. ISBN 0-8020-3765-8.

[9] Harper, J. A. (1995). "Samuel Kier - Medicine Man \& Refiner". Pennsylvania Geology. Oil Region Alliance of Business, Industry \& Tourism. 26 (1). Archived from the original (Excerpt from Yo-Ho-Ho and a Bottle of Unrefined Complex Liquid Hydrocarbons) on 15 March 2012.

[10] Steil, Tim; Luning, Jim (2002). Fantastic Filling Stations. MBI Publishing. ISBN 0-7603-1064-5.
[11] Cooley, Le Roy Clark (1873). Elements of Chemistry: for Common and High Schools. Scribner, Armstrong. p. 98.

[12] Crew, Benjamin Johnson; Ashburner, Charles Albert (1887). A Practical Treatise on Petroleum. Baird. pp. 395. This reference uses "benzene" in the obsolescent generic sense of a volatile hydrocarbon mixture, now called benzine, petroleum ether, ligroin, or naphtha, rather than the modern meaning of benzene as the specific aromatic hydrocarbon C6H6.

[13] Bettmann, Otto (1974). The Good Old Days - They Were Terrible! Random House. p. 34. ISBN 978-0-394-70941-3.

[14] Onyeneke C. C. (2015). Multivariate Approach to Partial Correlation Analysis: Science Journal of Applied Mathematics and Statistics. Vol. 3, No. 3, 2015, pp. 165-170. doi: 10.11648/j.sjams.20150303.20.

[15] Casmir Chidiebere Onyeneke, Chibuzor Eguzouwa, (2018). Assessment, Determination and Modeling Effects of Infrastructural Decay on Rental Values in Nigeria (Case Study of Ehimiri, Agbama and Isieke Housing Estates), Engineering Mathematics. Vol. 2, No. 1, 2018, pp. 12-20. doi: 10.11648/j.engmath.20180201.12

[16] Bradsher, Keith (2008). "Fuel Subsidies Overseas Take a Toll on U.S." New York Times. 